\title{
Motor Vehicle Emission Modeling and Software Simulation Computing for Roundabout in Urban City
}

\author{
Haiwei Wang, ${ }^{1}$ Huiying Wen, ${ }^{1}$ Feng You, ${ }^{1}$ Jianmin $X u,{ }^{1}$ and Hailin Kui ${ }^{2}$ \\ ${ }^{1}$ School of Civil Engineering and Transportation, South China University of Technology, Guangzhou 510640, China \\ ${ }^{2}$ College of Transportation, Jilin University, Changchun 130025, China \\ Correspondence should be addressed to Feng You; youfeng77@126.com
}

Received 16 July 2013; Revised 11 October 2013; Accepted 27 October 2013

Academic Editor: Wuhong Wang

Copyright (c) 2013 Haiwei Wang et al. This is an open access article distributed under the Creative Commons Attribution License, which permits unrestricted use, distribution, and reproduction in any medium, provided the original work is properly cited.

\begin{abstract}
In urban road traffic systems, roundabout is considered as one of the core traffic bottlenecks, which are also a core impact of vehicle emission and city environment. In this paper, we proposed a transport control and management method for solving traffic jam and reducing emission in roundabout. The platform of motor vehicle testing system and VSP-based emission model was established firstly. By using the topology chart of the roundabout and microsimulation software, we calculated the instantaneous emission rates of different vehicle and total vehicle emissions. We argued that Integration-Model, combing traffic simulation and vehicle emission, can be performed to calculate the instantaneous emission rates of different vehicle and total vehicle emissions at the roundabout. By contrasting the exhaust emissions result between no signal control and signal control in this area at the rush hour, it draws a conclusion that setting the optimizing signal control can effectively reduce the regional vehicle emission. The proposed approach has been submitted to a simulation and experiment that involved an environmental assessment in Satellite Square, a roundabout in medium city located in China. It has been verified that setting signal control with knowledge engineering and Integration-Model is a practical way for solving the traffic jams and environmental pollution.
\end{abstract}

\section{Introduction}

In the traditional traffic environmental management research field, in order to improve fuel efficiency and reduce emissions, varieties of static conservation and emissions reduction technologies have been widely studied and used, such as the static control testing technology of the vehicle fuel consumption and exhaust emissions on cars or engines [1]. Recently, researchers from different countries have begun to explore the relationship between the traffic activities and the vehicle fuel consumption and emissions. They focused on the study of reducing the vehicle fuel consumption and vehicle emissions by intelligent transportation information control. Compared to the conventional static control technology, such a new control strategy stands out for its significant characteristics on dynamic energy-saving, with focus on the dynamic management control of the vehicles and traffic flow. Practices have shown that this dynamic control strategy can serve the purpose of energy conservation and emissions reduction in traffic system. Moreover, it is especially suitable for the economic construction in China at present.

Generally, vehicle emission models can be divided into the macroregional emission model, the medium emission model, and the microscopic emission model according to the applicable scale and function. Because of the difference in applicable scale, the emission model structures are also different [2]; these structures of the emission model are shown in Table 1.

As an important part in traffic networks, an intersection is a place where multiple traffic flows converge. Therefore, the intersection has always become the bottleneck in the traffic network. The vehicles which run through the intersection often have to slow down, stop, and then speed up and during this process more gas emissions will be produced in the intersection. Thus, it is significant to take an insight into the characteristics of vehicle exhaust emissions at intersections 
TABLE 1: Classification of vehicle emission models.

\begin{tabular}{lccc}
\hline $\begin{array}{l}\text { Classification of emission } \\
\text { models }\end{array}$ & $\begin{array}{c}\text { Transportation } \\
\text { model /data }\end{array}$ & Model parameter & Emission model pattern \\
\hline Macroscopic emission model & Regional transportation model & $\begin{array}{c}\text { Average speed } \\
\text { (macroscopic paramet) }\end{array}$ & $\begin{array}{c}\text { Regional emission (speed } \\
\text { correction factor method) }\end{array}$ \\
$\begin{array}{l}\text { Medium } \\
\text { Emission model }\end{array}$ & $\begin{array}{c}\text { The transport model based on } \\
\text { equipment; behavior } \\
\text { distribution pattern }\end{array}$ & $\begin{array}{c}\text { Acceleration, speed, and } v / c \text { that } \\
\text { are based on the traffic facilities } \\
\text { (medium parameter) }\end{array}$ & $\begin{array}{c}\text { The emissions of entire network } \\
\text { based on the types of } \\
\text { equipment }\end{array}$ \\
Microscopic emission model & $\begin{array}{c}\text { Microscopic transport mode; } \\
\text { Driving cycle mode }\end{array}$ & $\begin{array}{c}\text { Vehicle operation data per } \\
\text { second }\end{array}$ & $\begin{array}{c}\text { Vehicle emissions } \\
\text { per second }\end{array}$ \\
\hline
\end{tabular}

over the entire traffic network. Moreover, there are also some relevant practical researches about reducing vehicle emission by signal control in foreign countries.

In this field, Tzeng and Chen [3] are among the foregoers who take in account the environmental factors in traditional traffic assignment, and they established the multiobjective traffic assignment model. Rilett and Benedek [4] used a simple two node two path network to evaluate the environmental improvement quantitatively by using the IVHS technology. Hallmark et al. [5] studied the effects on exhaust emissions by the method of intersection signal timing. Under the condition of different speed limits, Jackson et al. [6] analyzed the motor emissions on the highway. For the single point control of the intersections, Yoon et al. [7] calculated the optimal signal formula to reduce the queuing delay and exhaust emission effect. Pandian et al. [8] calculated and compared the traffic characteristics and the vehicle exhaust emissions between the ETC system and the conventional toll stations. Zhangb and Zietsmana [9] made a comparative analysis of the emission factor on these two strategies of vehicles idling in front of red light and the motor restart after flameout in the intersection.

In addition, some scholars used the existing emission models to calculate vehicle emissions directly and combined it with the city traffic signal control optimization. Hallmark and Guensler [10] used Measure model to prove the effect of the signal timing on the emissions of CO. Li et al. [11] used the computation formula of Mobile model and proposed an overall optimization scheme that uses the signal timing to reduce the emissions. In China, the optimization methods and the related researches are also conducted by Southeast University, Wuhan University of Technology, Beijing Jiaotong University, and Jilin University [12-18].

According to the study of vehicle emission test on the road and at the intersection both at home and abroad, it is evident that the researches on vehicle emission are studied extensively in recent years. At the same time, more attention is paid to the subject of how to reduce the vehicle exhaust pollution by traffic management and traffic control methods, especially by the method of traffic signal control. However, when it comes to the city road vehicle exhaust pollution problems, there seems to be a long way to go in theoretical analysis and methodology.

This paper is organized as follows. Following the introduction, the problem description of roundabout is presented in Section 2. Section 3 illustrates emission measure test of city road and Section 4 illustrates the microscopic emission model for motor vehicle based on VSP. In the Section 5, we introduce the optimizing transportation management and control laws for roundabout intersection with low carbon. Finally, Section 6 concludes the paper.

\section{The Problem Description of Roundabout: Congestion and Polluted}

2.1. Traffic Characteristics of Roundabout. Changchun (China) is a city which owns a smaller number of overpasses, and most of the road segments are connected with various intersections. At present, with the improvement of people's living standard in modern society and the remarkable increase of motor vehicle volume, all kinds of road segments which are connected with intersections are overloaded. In particular some roundabouts (such as Xinmin square, People Square, and Satellite Square) have failed to satisfy the traffic demands, and serious traffic congestions often occurred in rush hours. And furthermore, these intersections also become the traffic accident black-spots. Once a traffic accident or traffic blocks occurs at such spots, it would turn into a large obstacle to the whole traffic system and cause serious traffic environment pollution, which cuts down the traffic capacity of road network continuously.

In this paper, a typical roundabout, Satellite Square Roundabout, as is shown in Figure 1, is selected as the study site. It is an important transport hub throughout the northsouth and eastwest areas. With a circle radius of $70 \mathrm{~m}$, the roundabout is a landmark building in Changchun. And it is a large intersection of no traffic signal control, which means that traffic flows running through mingle together freely. The traffic characteristics in this intersection are manifested as follows.

(1) The mixed traffic flow of pedestrians and vehicles is unbalanced in time and space distributions. Thus, traffic congestion occurs frequently on the intersection, especially at the two-way junction on the People Road.

(2) According to a traffic survey of each intersection, the traffic flow through the satellite square intersection has far exceeded the threshold of the intersection capacity in rush hours. It implies that at the present the original design capacity of the intersection has 

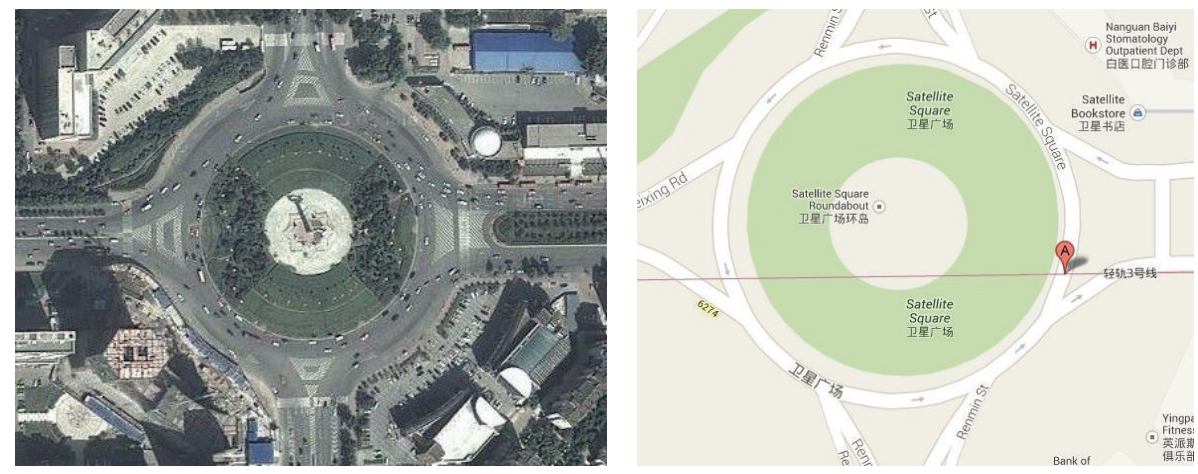

FIGURE 1: Satellite square roundabout aerial in Changchun.

failed to meet the growth of traffic demand in Changchun city.

(3) Taxi is a very important means of transportation in Changchun and occupies a large share of traffic flows. Furthermore, the operation and management mode of taxi enhances the probability of illegal driving activities. Also, the centralized distribution of bus stops near the Satellite Roundabout leads to large passenger flow and stagnation of the bus on the bus stop, which also exacerbates traffic conditions there.

(4) In the area of Satellite Roundabout, the coordination of intersection traffic flow is so poor, and it lacks drive auxiliary facilities, both of which contribute to the anomalies traffic flows on a certain crossroad. Moreover, unharmonious traffic flow of the intersection traffic eventually leads to serious traffic congestion and traffic pollution at the roundabout during the rush hour.

Therefore, this typical intersection (suffering from big saturation, serious traffic congestion, and serious motor exhaust pollution) serves the purpose of traffic pollution reduction research perfectly.

2.2. Traffic Control Scheme on the Roundabout. The key point of the intersection signal control is to assign a right-of-way for vehicles by signal light. Compared to intersections without signal control, stop lines are added on the imports of different lanes at the roundabout and signal lights with applicable signal timing are set up for the purpose of controlling vehicles running through. In this way, the traffic flows from different directions are separated in time dimension, which makes it possible to eliminate traffic congestion and problems of traffic pollution existing in the no signal control roundabout. The signal set analysis is shown in Figure 2. In a word, by optimizing the allocation of time and space resource, both the intersection traffic capacity and traffic order will be improved. Correspondingly, the traffic congestion and jams will decrease and the traffic efficiency will also be promoted.

\section{Emission Measure Test of City Road}

3.1. On-Board Emission Test System. In this paper, the onboard emission testing platform is chosen as the method for
The first phase in southnorth road

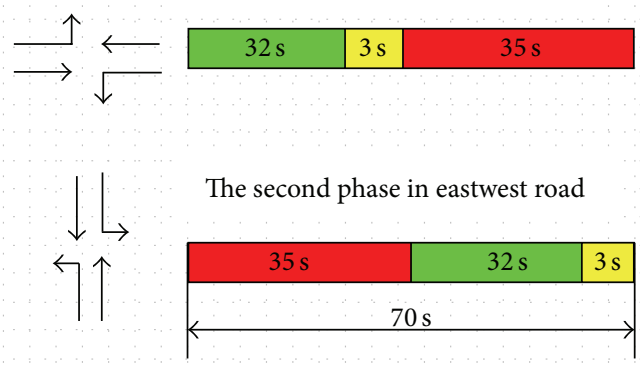

FIGURE 2: Signal set analysis.

test and Changchun city roads with different road grades are selected as the study object. Then, we can get the driving state of test vehicle going on the actual road and the corresponding emission test data per second, so as to pave the way for traffic area emissions analysis on the different traffic situations. In the experiment, we chose the portable car emissions measurement instrument OEM 2100 as the instrument for emission measurement. OEM 2100 is produced by Clean Air Technologies Inc of America and has passed the New York environmental protection department test in EPA's nation fuel and vehicle emission laboratory. For speed measurement, we chose TN-200GPS receiver (produced by Rayming and notebook computer) as real-time road speed measurement system. Its precision can reach 1 meter, and it can be placed at any position in the car, so it is a suitable subsidiary measuring instrument that measures vehicle driving condition on road motor experiment. Besides, we used GPS mapping software made by Fugawi Company to record vehicle road running parameters gathered by GPS. Actual on-road vehicle speed parameter, distance parameter, and other parameters are measured, and these data will be recorded in the computer. Figure 3 is the operation panel and the installation position in experiment of OEM-2100. Figure 4 is the structure diagram of the vehicle emission testing system.

3.2. Experimental Vehicle Selection. In accordance with "the measurement method of air pollution from motor vehicle 

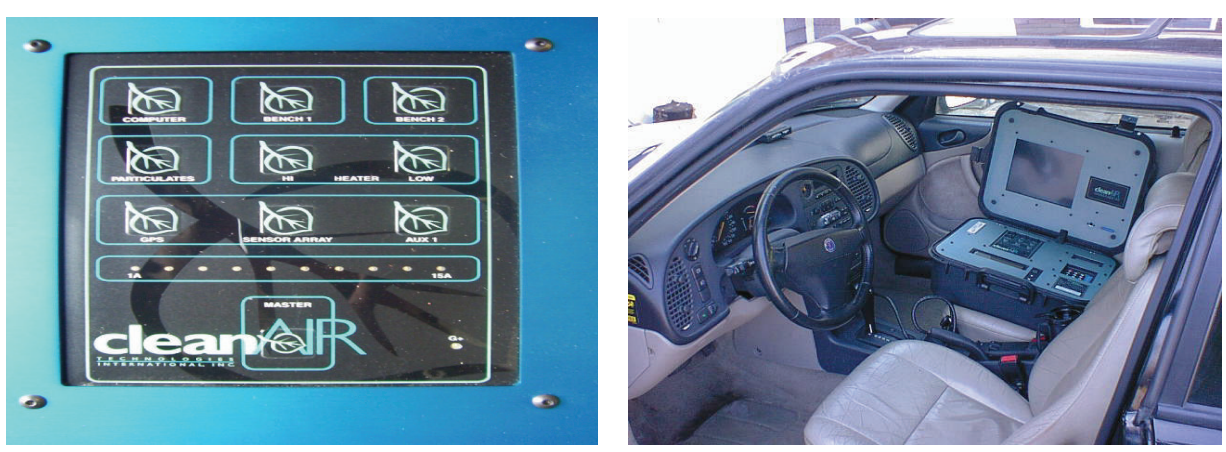

FIGURE 3: The operation panel and the installation position in experiment of OEM 2100.

TABLE 2: The test vehicle parameter statistics.

\begin{tabular}{lccccc}
\hline Vehicle type & Model & Productive life & Mileage & Engine displacement & Fuel supply \\
\hline \multirow{2}{*}{ Light vehicle } & Jetta Gix & 2001 & $42000 \mathrm{~km}$ & $1.6 \mathrm{~L}$ & Electric injection \\
& Red flag CA7201 & 2004 & $35000 \mathrm{~km}$ & $2.0 \mathrm{~L}$ & Electric injection \\
\multirow{2}{*}{ Midsize car } & Sea lion Ruichi & 2002 & $28000 \mathrm{~km}$ & $2.4 \mathrm{~L}$ & Electric injection \\
& Haf H3 & 2008 & $18000 \mathrm{~km}$ & $2.4 \mathrm{~L}$ & Electric injection \\
\hline
\end{tabular}
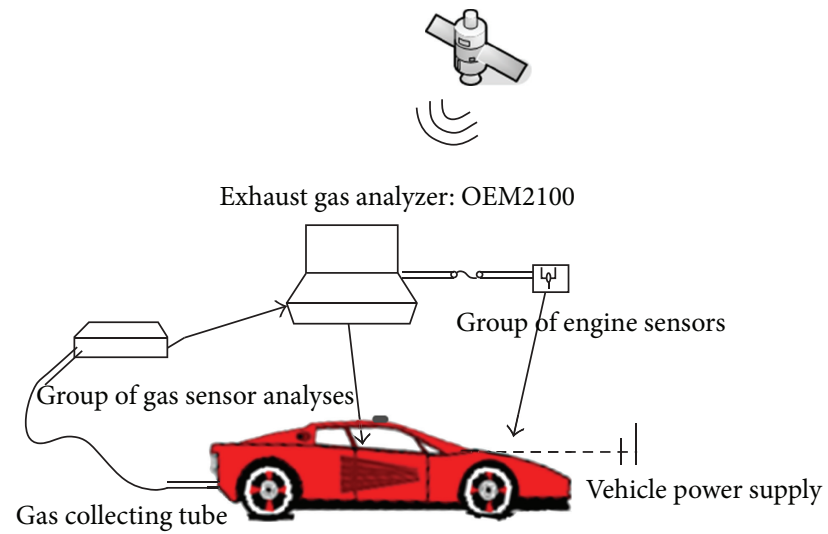

Figure 4: The structure of on-board emission test system.

emissions in urban" which is formulated by the State Environmental Protection Administration, research group conducted the emissions experiments of the on-road vehicle and the emissions calculation [19]. All test vehicles participated in the annual inspection on time per year and passed the test of pollution emissions. So the requirements of the road emission experiment are satisfied and the data we obtain are receivable. Besides, bus transportation is also included in the emission calculation. According to the measurement method, bus transportation (diesel vehicle) belongs to heavyduty vehicle. However, our test system can only measure emission data of light-duty gasoline vehicles, so there is no heavy-duty vehicle in the test experiment. As a result, we choose to refer to overseas and domestic research status when we set up emission model to calculate vehicle emissions.

The statistics data of the technology parameter of the test vehicle are as follows in the Table 2.
3.3. Experimental Road Selection. The road network pattern in Changchun city is a combination of a radioactive and irregular chessboard type, and commercial areas and administrative areas are both located in the center of city. We choose these typical city roads for experiment, as shown in Figure 5, for the reason that they bear the major traffic flows and are distinguished from each other in some aspects.

Path Line One. People Street (trunk road), Minkang Road (secondary trunk road), Jiefang Road (express way), Anda Street (secondary trunk road), Xi'an Road (express way), Changchun Street (express way), Yatai Street (Trunk Road), Nanhu Road (express way).

Path Line Two. North People Street, South People Street, Xi'an Road, Changchun Street, Minkang Road. Each road drives into People's Square Roundabout and each road drives out of People's Square Roundabout, and then drive around the People's Square Roundabout on the inner and outer ring road at normal speed.

3.4. The Emission Experiment Data Acquisition in Real-Time Road. By processing and matching experiment data, we can obtain the final database which is established from both the emission experiment data and vehicle driving data.

The experimental data processing flow chart is as shown in Figure 6.

\section{Microscopic Emission Model for Motor Vehicle Based on VSP}

4.1. Calculation Method of Vehicle Specific Power (VSP). In order to describe and calculate accurately the on-road motor vehicle emission pollution, Jose had proposed the concept of Vehicle Specific Power (VSP), he pointed out that driving 


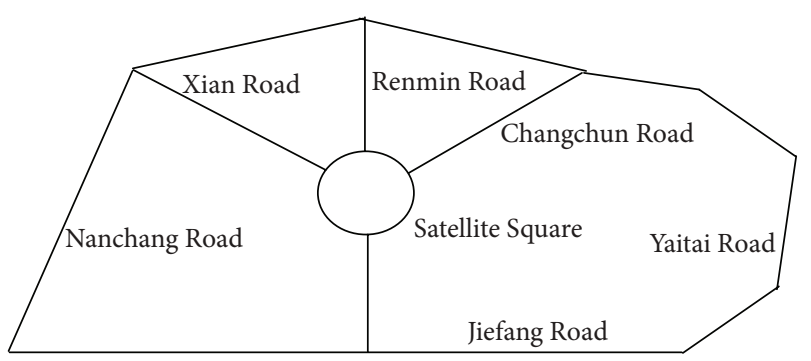

FIGURE 5: The road route of emission experiments.

conditions of motor vehicle have serious impact on these vehicle emission pollution.

According to the literature [20-23], vehicle specific power (VSP), with the unit of $\mathrm{kw} / \mathrm{t}$, is also referred to as instantaneous power per quality of motor vehicle. It consists of two parts. One is the output power of engine which is used for overcoming the friction from wheel rotation resistance, aerodynamics resistance and increasing kinetic, and potential energy of motor vehicle. And the other is transmission mechanical power loss caused by internal fingernail friction during transmission. As Figure 7 is the VSP of vehicle.

Mathematical expression (Jose, 1999a) is shown in [24]

$$
\mathrm{VSP}=\frac{(d(E K+P E) / d t)+F v+F_{A} v+F v_{i_{r}}}{m} ;
$$

formula expansion is

$$
\begin{aligned}
& \left(\left((d / d t)\left(0.5 \times m\left(1+\varepsilon_{i}\right) v^{2}+m g h\right)\right.\right. \\
& \left.\quad+C_{R} m g v+0.5 \times \rho_{a} C_{D} A\left(v+v_{m}\right)^{2} v+C_{i} m g v\right) \\
& \left.\quad \times(m)^{-1}\right) \\
& =v\left[a\left(1+\varepsilon_{i}\right)+g \times \sin \theta+g \times C_{R}\right] \\
& \quad+0.5 \times \rho_{a} \frac{C_{D} A}{m}\left(v+v_{m}\right)^{2} v+C_{i} g v,
\end{aligned}
$$

where $v$ is vehicle speed (in $\mathrm{m} / \mathrm{s}$ ); $m$ is vehicle weight (in $\mathrm{kg}$ ); $a$ is vehicle acceleration (in $\mathrm{m} / \mathrm{s}^{2}$ ); $\varepsilon_{i}$ is quality factor (nondimensional); $h$ is vehicle altitude (in $\mathrm{m}$ ); $\theta$ is road grade; $g$ is gravitational acceleration, $\left(9.81 \mathrm{~m} / \mathrm{s}^{2}\right) ; C_{D}$ is drag coefficient (nondimensional); $C_{R}$ is rolling resistance coefficient (nondimensional), which is connected with the type of tire and pavement materials; $A$ is vehicle windshield area (in $\left.\mathrm{m}^{2}\right) ; \rho_{a}$ is ambient air density $\left(1.207 \mathrm{~kg} / \mathrm{m}^{3}\right.$ at 20 centigrade); $v_{m}$ is wind speed; $C_{i}$ is internal friction coefficient (nondimensional), which is negligible.

Put the data measured directly into the formula, after simplifying the formula, we will get the following general formula of vehicle specific power:

$$
\mathrm{VSP}=v[1.1 a+9.8(a s)+0.132]+0.000302 v^{3},
$$

where $s=\tan (\sin \theta)$ is the gradient (nondimensional), which will be perceived as quotient of vehicle lift height and slope length when calculated.
TABLE 3: Ranges of VSP.

\begin{tabular}{lcc}
\hline VSP $(\mathrm{kw} / \mathrm{t})$ & Range & Frequency \\
\hline $\mathrm{VSP}<-10$ & 1 & 0.035 \\
$-10 \leq \mathrm{VSP}<2$ & 2 & 0.061 \\
$-2 \leq \mathrm{VSP}<0$ & 3 & 0.074 \\
$0=\mathrm{VSP}$ & 4 & 0.294 \\
$1 \leq \mathrm{VSP}<3$ & 5 & 0.212 \\
$3 \leq \mathrm{VSP}<5$ & 6 & 0.206 \\
$5 \leq \mathrm{VSP}<9$ & 7 & 0.037 \\
$9 \leq \mathrm{VSP}<13$ & 8 & 0.016 \\
$13 \leq \mathrm{VSP}<17$ & 9 & 0.016 \\
$17 \leq \mathrm{VSP}<20$ & 10 & 0.013 \\
$20 \leq \mathrm{VSP}$ & 11 & 0.037 \\
\hline
\end{tabular}

In order to show the load conditions of motor vehicles on-road, it is necessary to depict the driving conditions of moving vehicles precisely. Firstly, we figure out the correlation between instantaneous gas emissions of on-road vehicles and VSP and keep a track of driving conditions of on-road vehicle. Then on the basis of statistical probability value of VSP, we divided vehicle specific Power into eleven ranges [2527], as shown in the Table 3 . In the process of dividing, we adhere to the following two principles.

(1) When there are great fluctuations between different ranges, we will get these two different vehicle specific power frequency values into different ranges in order to calculate the different ranges of VSP.

(2) Dividing the vehicle specific power ranges as much as possible can help in reducing standard deviation of values of each range, as a result of which the computing result of the emissions will be more accurate.

4.2. The Microemission Model under VSP Model. Traditionally, researchers may use the average emission factors to calculate the actual pollutant emissions of road vehicles. However, such conventional methods generate large errors. Therefore, we now use actual road test to accurately describe and calculate the actual road vehicle emissions of gaseous pollutants situation.

Jose proposed road vehicle specific power for the first time, and he pointed out that emissions are affected by the probability of time throughout average speed and instantaneous speed of the vehicle and acceleration, and also affected by vehicle categories on-road.

According to the characteristics of the actual emissions of vehicles transient analysis, as is shown in Figure 8, we can know that for the actual road running vehicles, the instantaneous rate of discharge is significantly related to vehicle speed and specific power [28]. This correlation is even more evident in certain conditions of the running vehicles. According to the aforementioned specific power ranges, the research group established an actual road vehicles microscopic emissions model based on a large number of experimental data. 


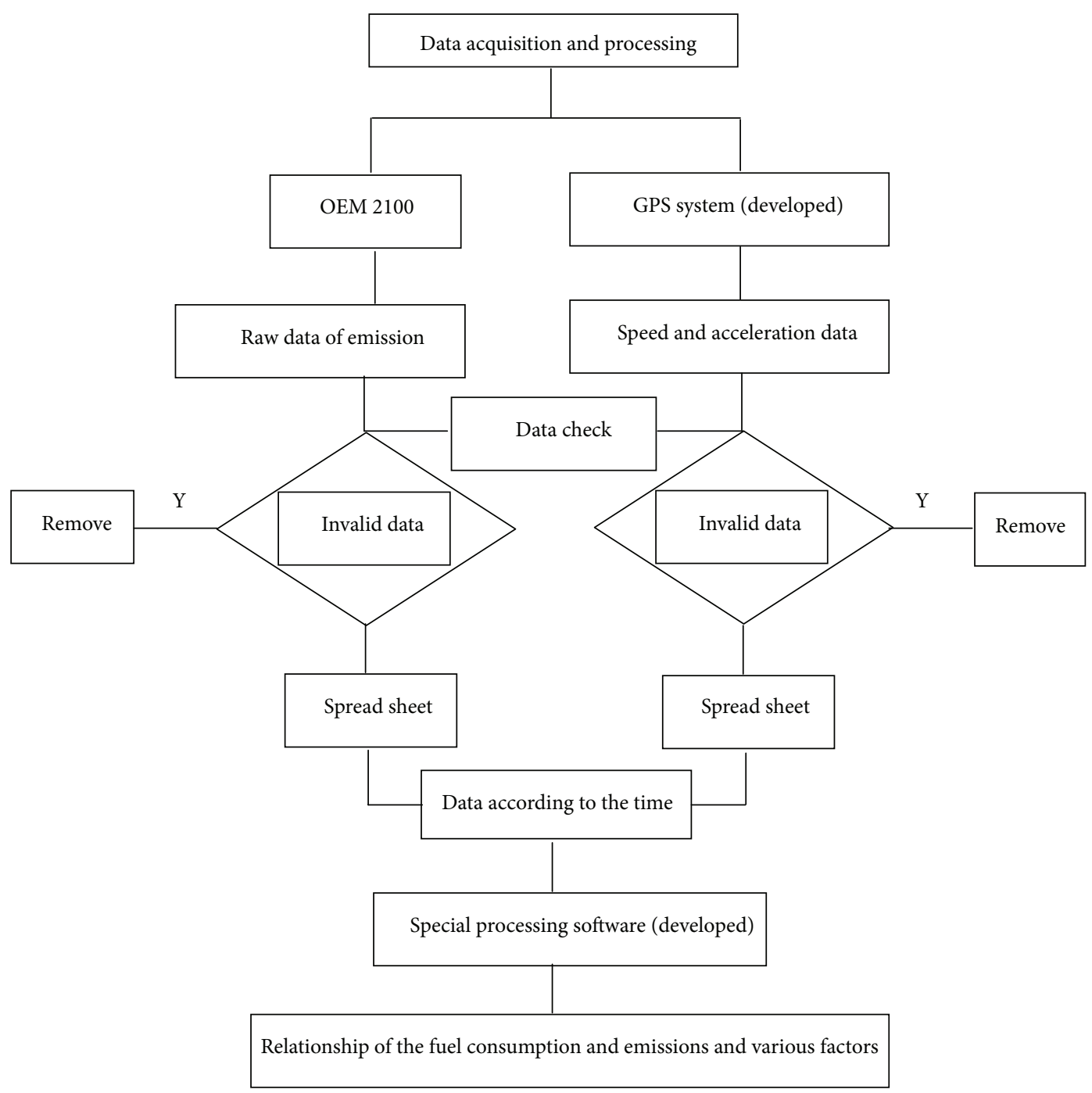

FIGURE 6: The processing flowchart for experimental data.

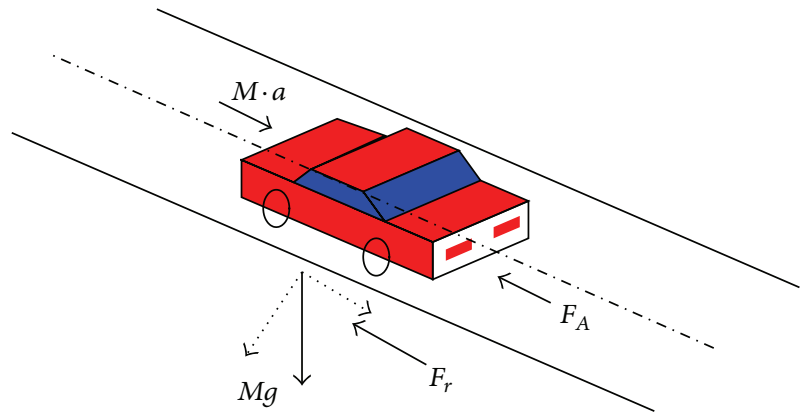

FIGURE 7: VSP of vehicle.

By analyzing driving condition data of on-road vehicle, we can get the required data of vehicle real-time speed and vehicle acceleration. Then, we import the data into VSP formula to generate the second-by-second data of VSP. In the next stage, these VSP values will be divided into different ranges, which then will be analyzed to develop a database.
According to the data characteristics, we could get the related statistical regular pattern in the way of regression analysis. On the basis of different probability distribution of the same VSP range, we calculate these 3 gas instantaneous vehicle emissions quality in each VSP range, as shown in Tables 4, 5 , and 6 Thus, the relationships between 11 VSP ranges and the 3 gas instantaneous vehicle emissions quality selected are formed, respectively. According to the analysis, VSP mostly spread over the ranges of $-3 \sim 7$, as shown in Figures 9, and 10.

\section{Optimizing Transportation Management and Control for Roundabout Intersection with Low Carbon}

In this section, a typical intersection suffering from traffic congestion is chosen as the object of the research. VISSIM (dynamic traffic simulation software) is used to simulate the real-time operation state. Then, the combining of VISSIM 


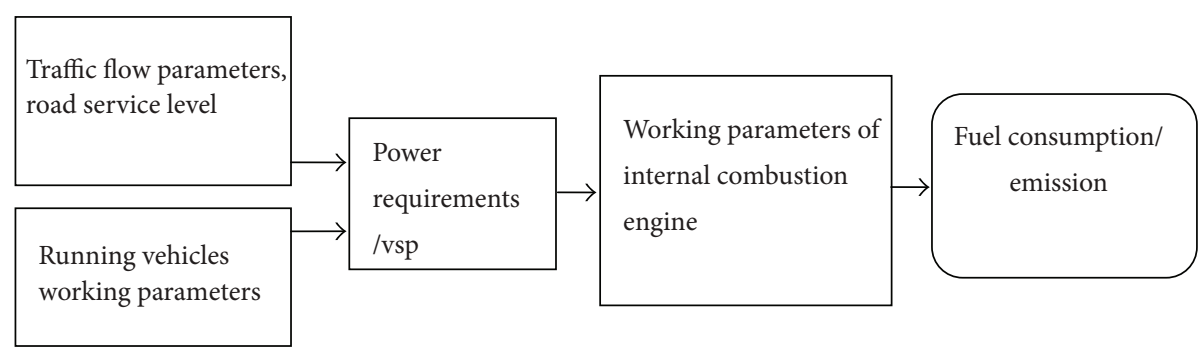

Figure 8: Microscopic emissions model flow of vehicles.

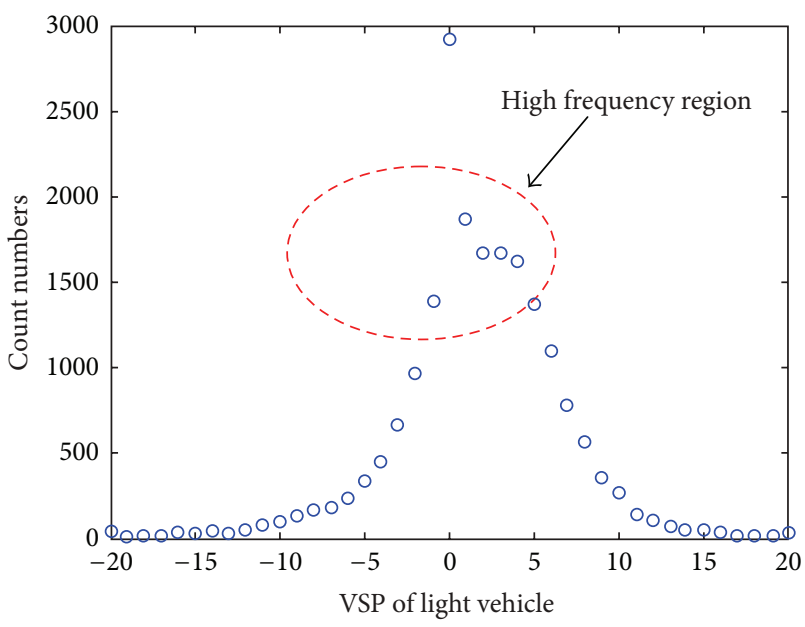

FIGURE 9: Specific power frequency distribution of light vehicles.

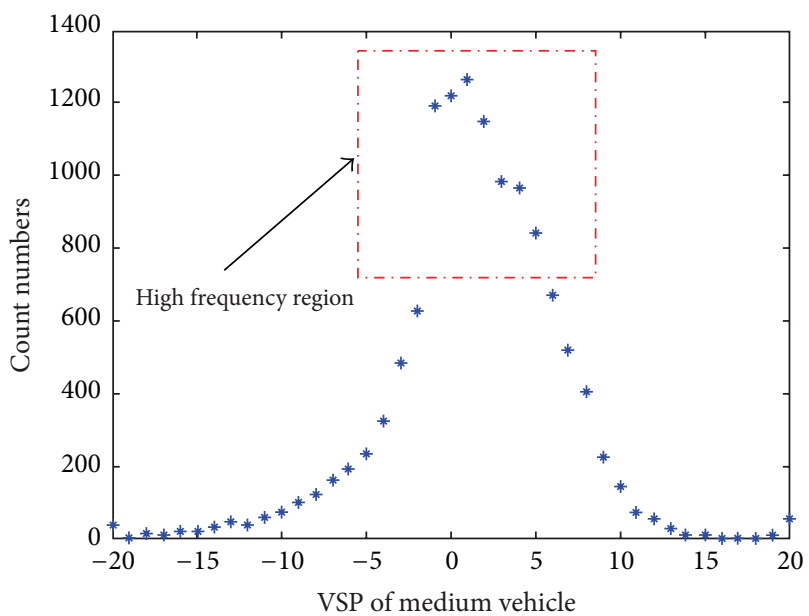

FIGURE 10: Specific power frequency distribution of middle vehicles.

with microscopic emission model can provide the necessary support for the emission of a certain traffic area. By calculating the vehicle driving emission of the chosen traffic area, we can find out approaches to improve the traffic situation. By the way, it also lays a foundation for energy conservation and emissions reduction, which can be achieved by optimizing the existing traffic control scheme.
TABLE 4: Rate of instantaneous vehicle emission quality under light vehicles specific power ranges.

\begin{tabular}{lcccc}
\hline Ranges of VSP & $\begin{array}{c}\text { Range of } \\
\text { values }\end{array}$ & $\mathrm{CO}(\mathrm{mg} / \mathrm{s})$ & $\mathrm{HC}(\mathrm{mg} / \mathrm{s})$ & $\mathrm{NOx}(\mathrm{mg} / \mathrm{s})$ \\
\hline 1 & $\mathrm{VSP}<-10$ & 1.9037 & 0.0659 & 0.3433 \\
2 & $-10 \leq \mathrm{VSP}<2$ & 2.0903 & 0.1032 & 0.5041 \\
3 & $-2 \leq \mathrm{VSP}<0$ & 2.5399 & 0.1589 & 0.5559 \\
4 & $0=\mathrm{VSP}$ & 1.9546 & 0.2456 & 0.5889 \\
5 & $1 \leq \mathrm{VSP}<3$ & 2.3780 & 0.1938 & 0.7026 \\
6 & $3 \leq \mathrm{VSP}<5$ & 2.2679 & 0.3001 & 0.8429 \\
7 & $5 \leq \mathrm{VSP}<9$ & 2.9534 & 0.3329 & 1.0572 \\
8 & $9 \leq \mathrm{VSP}<13$ & 4.0731 & 0.4380 & 1.1759 \\
9 & $13 \leq \mathrm{VSP}<17$ & 3.9956 & 0.5469 & 1.3584 \\
10 & $17 \leq \mathrm{VSP}<20$ & 4.5140 & 0.5177 & 1.4520 \\
11 & $20 \leq \mathrm{VSP}$ & 4.2339 & 0.5065 & 1.4531 \\
\hline
\end{tabular}

TABLE 5: Rate of instantaneous vehicle emission quality under middle-sized car vehicle specific power ranges.

\begin{tabular}{lcccc}
\hline Ranges of VSP & $\begin{array}{c}\text { Range of } \\
\text { values }\end{array}$ & CO $(\mathrm{mg} / \mathrm{s})$ & $\mathrm{HC}(\mathrm{mg} / \mathrm{s})$ & $\mathrm{NOx}(\mathrm{mg} / \mathrm{s})$ \\
\hline 1 & $\mathrm{VSP}<-10$ & 1.7339 & 0.2288 & 0.1237 \\
2 & $-10 \leq \mathrm{VSP}<2$ & 2.3420 & 0.2641 & 0.0899 \\
3 & $-2 \leq \mathrm{VSP}<0$ & 2.5245 & 0.2679 & 0.0767 \\
4 & $0=\mathrm{VSP}$ & 3.1301 & 0.3124 & 0.1695 \\
5 & $1 \leq \mathrm{VSP}<3$ & 3.4744 & 0.2699 & 0.1799 \\
6 & $3 \leq \mathrm{VSP}<5$ & 3.9401 & 0.3187 & 0.2536 \\
7 & $5 \leq \mathrm{VSP}<9$ & 5.0478 & 0.4341 & 0.3924 \\
8 & $9 \leq \mathrm{VSP}<13$ & 5.7706 & 0.5351 & 0.5249 \\
9 & $13 \leq \mathrm{VSP}<17$ & 5.5012 & 0.6296 & 0.5877 \\
10 & $17 \leq \mathrm{VSP}<20$ & 4.4231 & 0.6101 & 0.7433 \\
11 & $20 \leq \mathrm{VSP}$ & 4.0965 & 0.5921 & 0.7249 \\
\hline
\end{tabular}

5.1. The Establishment of the Roundabout Simulation Model. To establish the roundabout simulation model, it is necessary to know the characteristics of actual traffic area road data, traffic flow, and a specific time period. As shown in Figure 11 and Table 7. Then, according to the basic steps of simulation model, the aforementioned data are added to the model as parameters. 
TABLE 6: Rate of instantaneous vehicle emission quality under bus specific power ranges.

\begin{tabular}{lcccc}
\hline Ranges of VSP & $\begin{array}{c}\text { Range of } \\
\text { values }\end{array}$ & CO $(\mathrm{mg} / \mathrm{s})$ & $\mathrm{HC}(\mathrm{mg} / \mathrm{s})$ & $\mathrm{NOx}(\mathrm{mg} / \mathrm{s})$ \\
\hline 1 & $\mathrm{VSP}<-10$ & 0.3428 & 0.8936 & 2.3350 \\
2 & $-10 \leq \mathrm{VSP}<2$ & 0.8659 & 1.2138 & 3.8250 \\
3 & $-2 \leq \mathrm{VSP}<0$ & 3.3380 & 1.7685 & 12.293 \\
4 & $0=\mathrm{VSP}$ & 4.6788 & 1.9398 & 19.076 \\
5 & $1 \leq \mathrm{VSP}<3$ & 6.9784 & 1.9929 & 22.7980 \\
6 & $3 \leq \mathrm{VSP}<5$ & 7.7868 & 1.9834 & 23.8749 \\
7 & $5 \leq \mathrm{VSP}<9$ & 8.5160 & 1.9482 & 24.209 \\
8 & $9 \leq \mathrm{VSP}<13$ & 7.9499 & 2.0052 & 26.620 \\
9 & $13 \leq \mathrm{VSP}<17$ & 6.5076 & 2.1758 & 27.345 \\
10 & $17 \leq \mathrm{VSP}<20$ & 6.2015 & 2.1810 & 30.827 \\
11 & $20 \leq \mathrm{VSP}$ & 5.2770 & 2.2033 & 32.970 \\
\hline
\end{tabular}

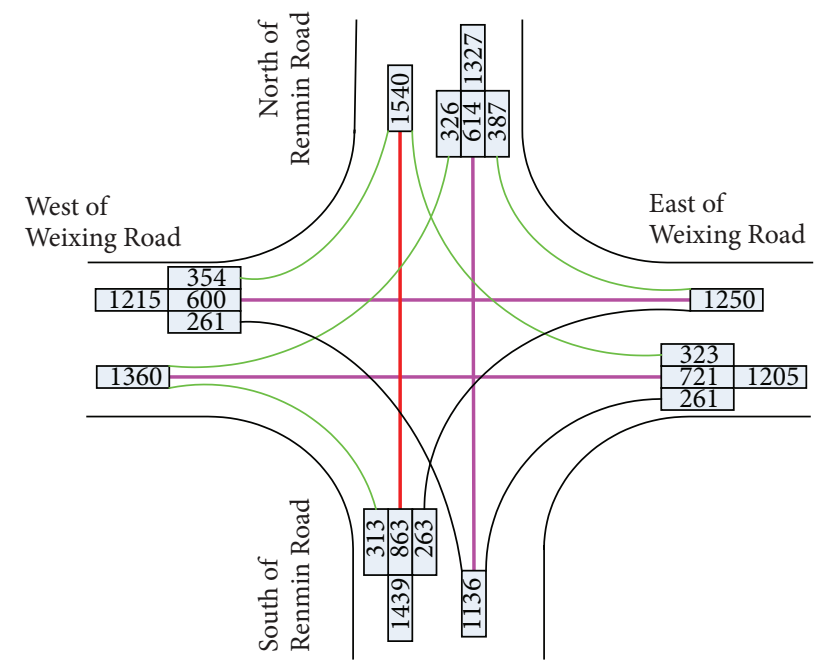

FIGURE 11: The traffic flow graph of intersection at peak hour.

On the basis of the traffic survey data, the motor vehicle flow at the intersection is about $5000 \mathrm{pcu} / \mathrm{h}$. Additionally, the intersection is located at the confluence of two trunk roads and pedestrians flow is large. As a result, the capability of entrance roads without intersection controls cannot satisfy the increasing traffic demand, which becomes the traffic bottlenecks.

5.2. Vehicle Emissions Compute and Simulation Model for Roundabout. After establishing the typical intersection traffic area by microscopic traffic simulation software, we can obtain the vehicle's instantaneous speed and acceleration in any single area of simulation. Then, by combining with micro emissions model based on VSP, we can calculate the motor vehicle emissions of intersection area and car emissions share rate, which could serve the purpose of the evaluation of traffic environment.

We get the integer data based on VSP and put them into different 11 VSP ranges for statistical compute. With the emission model, we get the average of the transient emissions rate from different types of vehicles in traffic simulation area, which is shown in Table 8. The following table shows the average rate of instantaneous vehicle emission quality.

Depending on the average rate of instantaneous vehicle emission quality as well as the total running time of the driving vehicle in the simulation, we can calculate the three kinds of different vehicle pollutant emissions in the morning rush for one hour.

For a particular intersection traffic simulation area, the calculation formula of the total toxic emission is as follows:

$$
G_{E j}=\sum_{i=1}^{m} \sum_{j=1}^{n} G\left(p_{i j}\right) M\left(p_{i j}\right),
$$

where $G_{E j}$ is total vehicle emissions of a certain vehicle; $J$ represents three vehicle models (light, medium, and heavy); $i$ is for different VSP ranges; $p_{i j}$ is for the $j$ models in VSP range of the $i ; G\left(p_{i j}\right)$ means the cumulative time s of the $j$ models in VSP range $i ; M\left(p_{i j}\right)$ is for quality of emissions of the $j$ models in VSP range $i, \mathrm{~g} / \mathrm{s}$.

The total emissions in the whole area can be calculated by the following formula:

$$
A=\sum_{j=1}^{n} G_{E j},
$$

$A$ is the total regional emissions. Table 9 is total gas pollutants emissions in an hour in simulation area.

5.3. Gas Emission Calculate Result of the Roundabout under Traffic Signal Control. Roundabout is an important part of city roads, and its capacity largely depends on how well an intersection traffic flow runs. Traffic capacity of Roundabout is limited by the weaving section capacity. In the case of a traffic increase in particular, when the traffic flow gets close to or exceeds the traffic capacity of roundabout, it can cause ring road traffic congestion. And then the vehicle emissions pollution exceeds amount in the intersection area.

To achieve a reasonable allocation on time and space of traffic flow in traffic areas by setting up traffic signal light and signal timing optimization at the entrance of the roundabout, we can not only balance the entrance traffic flow but also eliminate the traffic congestion at the entrance, which reduces the vehicle delay in the traffic area. As shown in Figure 12.

By adding traffic signal lights and optimizing signal timing, we get the instantaneous speed and acceleration of each single vehicle from simulation software and calculate the single vehicle's VSP. Then we put VSP data into model to calculate so that we can get instantaneous emission rate and total quality of these three kinds of gas emissions in one hour. We put these data to calculate instantaneous gas emissions at the roundabout with traffic signals for different vehicle models and calculate the whole intersection of gas emissions. Results are shown in Tables 10 and 11.

5.4. Comparison of Environmental Protection Quota for Roundabout under Signal Control or under No Signal Control. By setting up traffic signal light and optimizing the signal timing at the roundabout, it can be found that the 
TABLE 7: Intersection road structure and traffic data.

\begin{tabular}{lcccccc}
\hline \multirow{2}{*}{ Entrance name } & \multicolumn{2}{c}{ Lane number $*$ width $(\mathrm{m})$} & \multicolumn{2}{c}{ Steering vehicle volume percentage (\%) } & \multirow{2}{*}{ Traffic flow (Pcu/h) } & \multirow{2}{*}{ Midsize cars } \\
& & Left & Straight & Right & & \\
\hline People's avenue north & $3 \times 3.2$ & 21 & 56 & 23 & 1540 & $13 \%$ \\
People's avenue south & $3 \times 3.2$ & 23 & 54 & 23 & 23 & 136 \\
Satellite Road left & $3 \times 2.6$ & 24 & 53 & 31 & 1360 & 1250 \\
Satellite Road east & $3 \times 2.6$ & 21 & 48 & & $13 \%$ \\
\hline
\end{tabular}

TABLE 8: Average of rate of instantaneous vehicle emission quality for different models.

\begin{tabular}{lcccc}
\hline $\begin{array}{l}\text { Gas } \\
\text { (discharge rate) }\end{array}$ & Light vehicle & Midsize vehicle & Bus & Sum $(\mathrm{mg} / \mathrm{s})$ \\
\hline NOx (mg/s) & 0.6066 & 0.2325 & 21.029 & 21.8681 \\
CO (mg/s) & 2.3011 & 3.3060 & 4.0993 & 9.7064 \\
HC (mg/s) & 0.2400 & 0.3706 & 1.9075 & 2.5181 \\
\hline
\end{tabular}

TABLE 9: Total gas pollutants emissions in an hour in simulation area.

\begin{tabular}{lcccc}
\hline $\begin{array}{l}\text { Gas pollutants } \\
(\mathrm{kg} / \mathrm{h})\end{array}$ & \multicolumn{5}{c}{$\begin{array}{c}\text { Models } \\
\text { Light vehicle }\end{array}$} & Midsize vehicle & Bus & Sum $(\mathrm{kg} / \mathrm{h})$ \\
\hline NOx & 9.1717 & 0.837 & 2.2711 & 12.2798 \\
$\mathrm{CO}$ & 34.7926 & 11.9016 & 0.4427 & 47.1369 \\
$\mathrm{HC}$ & 3.6288 & 1.3341 & 0.2060 & 5.1689 \\
$\begin{array}{l}\text { Vehicle } \\
\text { emissions }\end{array}$ & 47.5931 & 14.0727 & 2.9198 & 64.5856 \\
\hline
\end{tabular}

TABLE 10: Instantaneous gas emissions at the roundabout under traffic signals for different vehicle models.

\begin{tabular}{lccc}
\hline Gas $(\mathrm{mg} / \mathrm{s})$ & Light vehicle & $\begin{array}{c}\text { Models } \\
\text { Midsize vehicle }\end{array}$ & Bus \\
\hline CO & 2.1123 & 3.0012 & 3.5688 \\
HC & 0.2036 & 0.3298 & 1.6970 \\
NOx & 0.5798 & 0.2175 & 19.1548 \\
\hline
\end{tabular}

TABLE 11: Gas emissions at the roundabout under traffic signals.

\begin{tabular}{lcc}
\hline Gas pollutant & Emissions from all sources $(\mathrm{kg} / \mathrm{h})$ & Percentage (\%) \\
\hline CO & 42.3289 & 73.12 \\
HC & 10.7791 & 18.62 \\
NOx & 4.7843 & 8.26 \\
\hline
\end{tabular}

vehicle under the traffic control is in a relatively steady state, as shown in the simulation output of road vehicle running status, we can get the comparisions of different vehicle's instantaneous gas emission rate in Tables 12, 13 and 14. During the rush hour, intersection congestion rarely appears at the entrance, which means that the traffic flow is in smooth running state, and the times of acceleration and deceleration of a single vehicle reduce which makes the transient emissions rate of gas pollutant decline obviously.

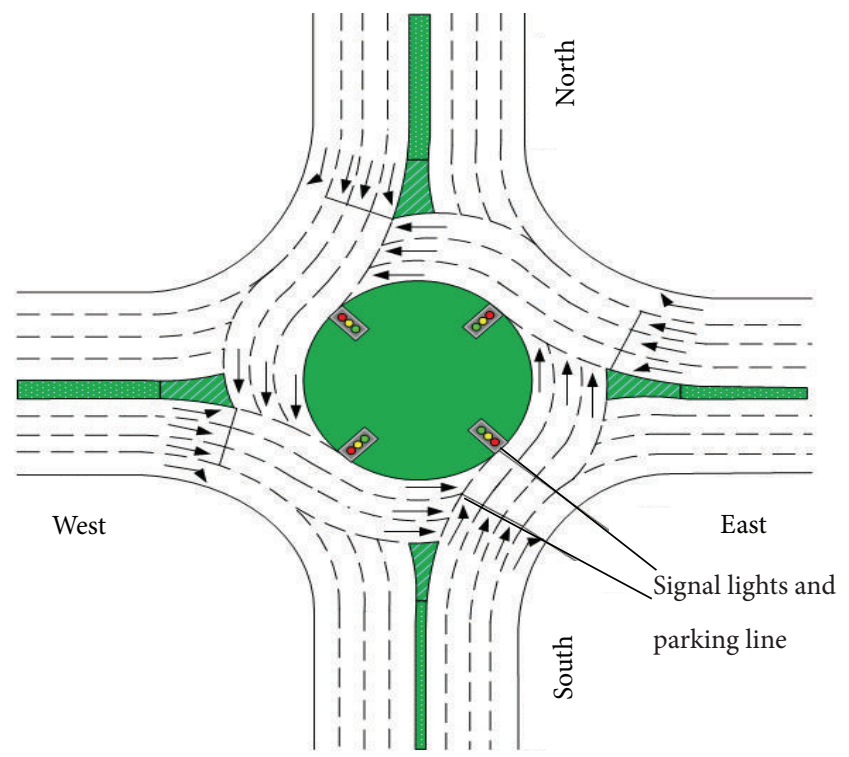

Figure 12: Setting signals at the roundabout.

TABLE 12: Comparison of light vehicle's instantaneous gas emissions rate.

\begin{tabular}{lccc}
\hline Control & \multicolumn{3}{c}{ Gas $(\mathrm{mg} / \mathrm{s})$} \\
& $\mathrm{CO}$ & $\mathrm{HC}$ & $\mathrm{NOx}$ \\
\hline In control & 2.3011 & 0.2400 & 0.6066 \\
No control & 2.1123 & 0.2036 & 0.5798 \\
Improved percentage & $8.20 \%$ & $15.16 \%$ & $4.42 \%$ \\
\hline
\end{tabular}

5.5. Contrastive Analysis of the Traffic Flow Indicators at the Roundabout Based on Simulation. We get some important evaluation indexes by VISSIM 3.60. Because at the beginning of the simulation the traffic needs a certain amount of time to stabilize, this period of time must be excluded from the simulation time of 3600s when we make the evaluation for a variety of traffic parameters $[17,18,29]$. That means we make the data statistics with the output data at the time of a relatively stable traffic flow. To get a better understanding of the test, we compare two kinds of states at the roundabout and the evaluation indexes are shown in the following Tables 15 and 16, and Figure 13. 


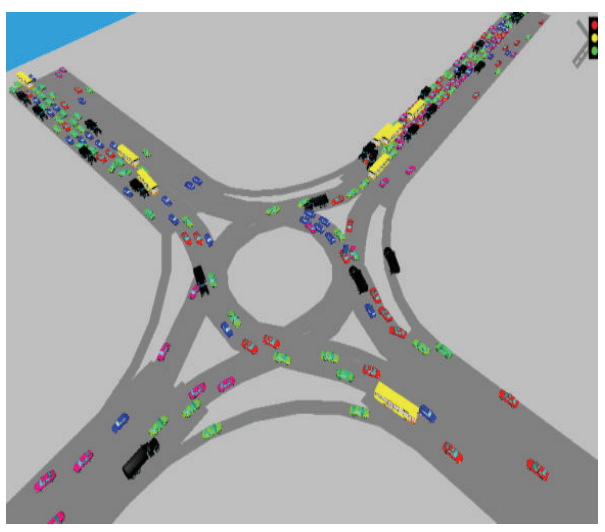

(a) No control

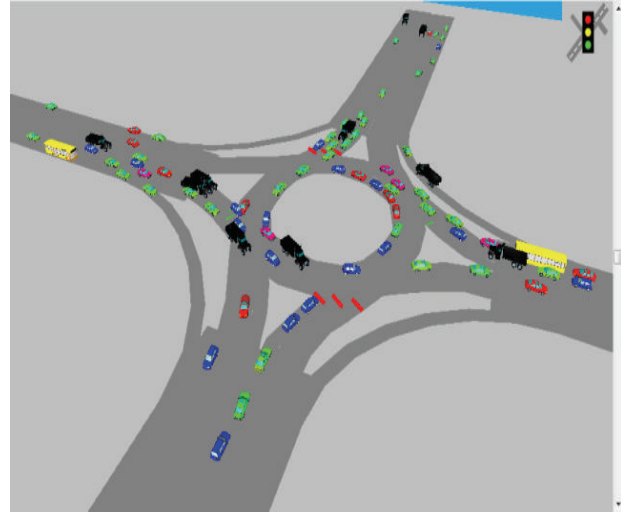

(b) In control

FIGURE 13: Roundabout with control VS roundabout without control.

TABLE 13: Comparison of midsize vehicle's instantaneous gas emissions rate.

\begin{tabular}{lccc}
\hline Control & \multicolumn{3}{c}{ Gas $(\mathrm{mg} / \mathrm{s})$} \\
& $\mathrm{CO}$ & $\mathrm{HC}$ & $\mathrm{NOx}$ \\
\hline No control & 3.306 & 0.3706 & 0.2325 \\
In control & 3.0012 & 0.3298 & 0.2175 \\
Improved percentage & $9.22 \%$ & $11.00 \%$ & $6.45 \%$ \\
\hline
\end{tabular}

TABLE 14: Comparison of bus's instantaneous gas emissions rate.

\begin{tabular}{lccc}
\hline Control & \multicolumn{3}{c}{ Gas $(\mathrm{mg} / \mathrm{s})$} \\
& $\mathrm{CO}$ & $\mathrm{HC}$ & $\mathrm{NOx}$ \\
\hline No control & 4.0993 & 1.9075 & 21.0290 \\
In control & 3.5688 & 1.6970 & 19.1548 \\
Improved percentage & $12.94 \%$ & $11.04 \%$ & $8.91 \%$ \\
\hline
\end{tabular}

\section{Conclusion}

Choose the car exhaust gas detection equipment (MONTATA OEM 2100) certificated by the U.S. environmental protection agency (EPA) and Vehicle driving condition monitor system based on GPS to construct the motor-mounted data acquisition system for emission test and make data acquisition for on-road vehicle driving condition and vehicle exhaust second-by second test. Through theoretical analysis of VSP and a large number of theoretical researches both at home and abroad, we use VSP which includes on-road vehicle instantaneous velocity, acceleration, and specific power of road slope as a modeling basis.

We use the database from the experiments and basic principle of vehicles specific power and the reference standard of the on-road driving situation to get the motor vehicle actual road micro emissions model based on three different vehicle models. At the same time, we get rate of instantaneous vehicle emission quality in the different VSP ranges. Thus, combined with the microsimulation model we can evaluate the traffic environment in the certain traffic region based on the Microscopic emission model.
TABLE 15: Comparison of traffic average volume.

\begin{tabular}{lccc}
\hline Control & $\begin{array}{c}\text { Light vehicle } \\
(\mathrm{km} / \mathrm{h})\end{array}$ & $\begin{array}{c}\text { Models } \\
\text { Midsize vehicle } \\
(\mathrm{km} / \mathrm{h})\end{array}$ & Bus $(\mathrm{km} / \mathrm{h})$ \\
\hline No control & 12.36 & 12.77 & 8.90 \\
In control & 14.11 & 14.94 & 9.83 \\
Percentage (\%) & 14.16 & 16.99 & 10.45 \\
\hline
\end{tabular}

TABLE 16: Comparison of traffic operation.

\begin{tabular}{|c|c|c|c|}
\hline \multirow{2}{*}{ Control } & \multicolumn{3}{|c|}{ Indicators } \\
\hline & $\begin{array}{c}\text { Average } \\
\text { delay (s/veh) }\end{array}$ & $\begin{array}{l}\text { Average number of } \\
\text { parking (times/veh) }\end{array}$ & Saturation \\
\hline No control & 91.4 & 4.69 & 1.42 \\
\hline In control & 39.7 & 2.57 & 0.83 \\
\hline Improved percentage & $56.5 \%$ & $45.2 \%$ & $41.5 \%$ \\
\hline
\end{tabular}

The results of the experiment show that under the traffic signal control the total gas emissions of $\mathrm{CO}, \mathrm{HC}$, and NOX for the same flow all reduced but in different degrees, with CO by $10.20 \%$, HC by $12.22 \%$, and NOX by $7.44 \%$. When it comes to pollutant instantaneous gas emissions rate of different vehicle models, we can find that $\mathrm{HC}$ gas emissions show the biggest drop in light vehicle, and the NOX gas emissions reduce most in bus. In summary, it shows that with the increment of traffic flow, setting traffic signal control at the roundabout can reduce traffic pollution emissions of the region effectively. In addition, when putting optimized timing parameter into the simulation software VISSIM, we got the evaluation parameters of traffic flow, including the average delay and average number of parking, saturation, and so forth. By comparing the simulation results (which are mainly related to traffic flow and traffic environment evaluation) under both conditions (with signal control and without signal control at the roundabout), we may deduce that under the traffic signal control, whether there is a rush hour or not, both the instantaneous vehicle emission quality and total traffic gas pollutant emissions are reduced to a certain extent. 
At the same time, corresponding traffic flow status is also improved and regional traffic congestion is alleviated.

In summary, this paper provided a new idea and technology investigation about traffic environment evaluation for a particular area under traffic control.

\section{Conflict of Interests}

The authors declare that there is no conflict of interests regarding the publication of this article.

\section{Acknowledgments}

This work was partially supported by the National Natural Science Foundation of China (Granted nos. 51378222, 51108192 and 51208500), Chinese Postdoctoral Science Foundation (the Granted no. 2012M521824 and no. 2013T60904). and the Fundamental Research Funds for the Central University of China (Granted no.: 2012ZZ0100). The first author would like to appreciate Dr. Feng You and Professor Wen Huiying, for the valuable discussions to improve the quality and presentation of the paper.

\section{References}

[1] H. Liu and M. Barth, "Identifying the effect of vehicle operating history on vehicle running emissions," Atmospheric Environment, vol. 2, no. 5, pp. 873-879, 2012.

[2] S. H. Cousins, J. Garcia Bueno, and O. Palomares Coronado, "Powering or de-powering future vehicles to reach low carbon outcomes: the long term view 1930-2020," Journal of Cleaner Production, vol. 15, no. 11-12, pp. 1022-1031, 2007.

[3] G.-H. Tzeng and C.-H. Chen, "Multiobjective decision making for traffic assignment," IEEE Transactions on Engineering Management, vol. 40, no. 2, pp. 180-187, 1993.

[4] L. R. Rilett and C. M. Benedek, "Traffic assignment under environmental and equity objectives," Transportation Research Record, no. 1443, pp. 92-99, 1994.

[5] S. L. Hallmark, I. Fomunung, R. Guensler, and W. Bachman, "Assessing impacts of improved signal timing as a transportation control measure using an activity-specific modeling approach," Transportation Research Record, vol. 1738, pp. 49-55, 2000.

[6] E. Jackson, Y. Qu, B. A. Holmén, and L. Aultman-Hall, “Driver and road type effects on light-duty gas and particulate emissions," Transportation Research Record, vol. 1987, pp. 118-127, 2006.

[7] S. H. Yoon, J. P. Cha, and C. S. Lee, "An investigation of the effects of spray angle and injection strategy on dimethyl ether (DME) combustion and exhaust emission characteristics in a common-rail diesel engine," Fuel Processing Technology, vol. 91, no. 11, pp. 1364-1372, 2010.

[8] S. Pandian, S. Gokhale, and A. K. Ghoshal, "Evaluating effects of traffic and vehicle characteristics on vehicular emissions near traffic intersections," Transportation Research D, vol. 14, no. 3, pp. 180-196, 2009.

[9] J. Lv, Y. Zhangb, and J. Zietsmana, "Investigating emission reduction benefit from intersection signal optimization," Journal of Intelligent Transportation Systems, vol. 17, no. 3, pp. 200209, 2013.
[10] S. L. Hallmark and R. Guensler, "Comparison of speedacceleration profiles from field data with NETSIM output for modal air quality analysis of signalized intersections," Transportation Research Record, no. 1664, pp. 40-46, 1999.

[11] X. Li, G. Li, S.-S. Pang, X. Yang, and J. Tian, "Signal timing of intersections using integrated optimization of traffic quality, emissions and fuel consumption: a note," Transportation Research D, vol. 9, no. 5, pp. 401-407, 2004.

[12] K. Chen and L. Yu, "Microscopic traffic-emission simulation and case study for evaluation of traffic control strategies," Journal of Transportation Systems Engineering and Information Technology, vol. 7, no. 1, pp. 93-99, 2007.

[13] P. Jiao and H. Lu, "Study on evaluation of national economic benefit of urban traffic manager," Journal of Highway and Transportation Research and Development, vol. 9, article 32, 2005.

[14] F. Mo, L. Yu, and G. H. Song, "An overview of portable emissions measurement system technologies and relevant research," Vehicle \& Power Technology, vol. 4, article 13, 2006.

[15] S.-X. Guo, L. Yu, and G. H. Song, "A comparative analysis of real-world emission factors and MOBILE6 predictive value for a heavy-duty diesel vehicle," Safety and Environmental Engineering, vol. 14, no. 2, pp. 17-21, 2007.

[16] K. Chen and L. Yu, "Microscopictraffic-emission simulation and case study for evaluation of traffic control strategies," Journal of Transportation Systems Engineering and Information Technology, vol. 7, no. 1, pp. 93-99, 2007.

[17] W. Wang, X. Jiang, S. Xia, and Q. Cao, "Incident tree model and incident tree analysis method for quantified risk assessment: an in-depth accident study in traffic operation," Safety Science, vol. 48, no. 10, pp. 1248-1262, 2010.

[18] C. Ding, W. Wang, X. Wang, and M. Baumann, "A neural network model for driver's lane-changing trajectory prediction in urban traffic flow," Mathematical Problems in Engineering, vol. 2013, Article ID 967358, 9 pages, 2013.

[19] D. Wan, J. Xu, J. Zhang, X. Tong, and J. Hu, "Historical and projected emissions of major halocarbons in China," Atmospheric Environment, vol. 43, no. 36, pp. 5822-5829, 2009.

[20] G. Song, L. Yu, and Z. Tu, "Distribution characteristics of vehicle-specific power on urban restricted-access roadways," Journal of Transportation Engineering, vol. 138, no. 2, pp. 202209, 2011.

[21] M. C. Coelho, H. C. Frey, N. M. Rouphail, H. Zhai, and L. Pelkmans, "Assessing methods for comparing emissions from gasoline and diesel light-duty vehicles based on microscale measurements," Transportation Research D, vol. 14, no. 2, pp. 9199, 2009.

[22] P. Zavadinka, "Simulation of vehicle transport duty cycle with using of hydrostatic units control algorithm," in Mechatronics, pp. 395-402, Springer, 2012.

[23] E. Demir, T. Bektaş, and G. Laporte, "A comparative analysis of several vehicle emission models for road freight transportation," Transportation Research D, vol. 16, no. 5, pp. 347-357, 2011.

[24] R. Casati, V. Scheer, R. Vogt, and T. Benter, "Measurement of nucleation and soot mode particle emission from a diesel passenger car in real world and laboratory in situ dilution," Atmospheric Environment, vol. 41, no. 10, pp. 2125-2135, 2007.

[25] Y. Wang, D. Guo, S. Li et al., "Research on urban intersection vehicle emission reduction based on microcosmic simulation," in Proceedings of the 9th International Conference of Chinese Transportation Professionals, pp. 2068-2074, Harbin, China, August 2009. 
[26] H. Huo, Y. Wu, and M. Wang, "Total versus urban: well-towheels assessment of criteria pollutant emissions from various vehicle/fuel systems," Atmospheric Environment, vol. 43, no. 10, pp. 1796-1804, 2009.

[27] Y. Wang, D. Guo, S. Li, Y. Wang, and Z. Li, "Signal timing optimization simulation on urban road intersection based on vehicle emissions," in Proceedings of the 8th International Conference of Chinese Logistics and Transportation ProfessionalsLogistics, pp. 4762-4767, Chengdu, China, August 2008.

[28] W. Wuhong, Z. Wei, and B. Herner, "Car-following safety algorithms based on adaptive cruise control strategies," in Proceedings of the 5th International Symposium on Intelligent Systems and Informatics, pp. 135-140, Subotica, Serbia, August 2007.

[29] W. Wang, W. Zhang, H. Guo, H. Bubb, and K. Ikeuchi, "A safetybased approaching behavioural model with various driving characteristics," Transportation Research C, vol. 19, no. 6, pp. 1202-1214, 2011. 


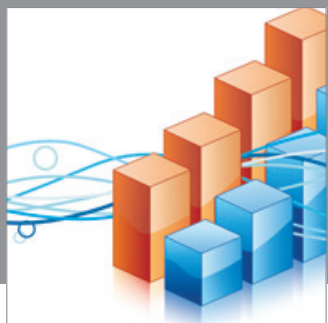

Advances in

Operations Research

mansans

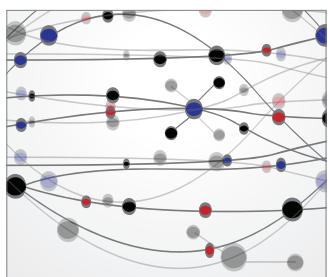

The Scientific World Journal
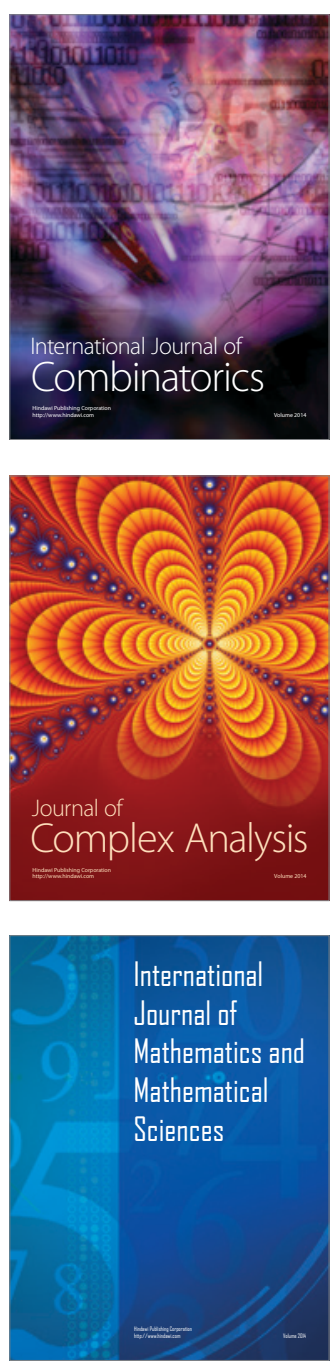
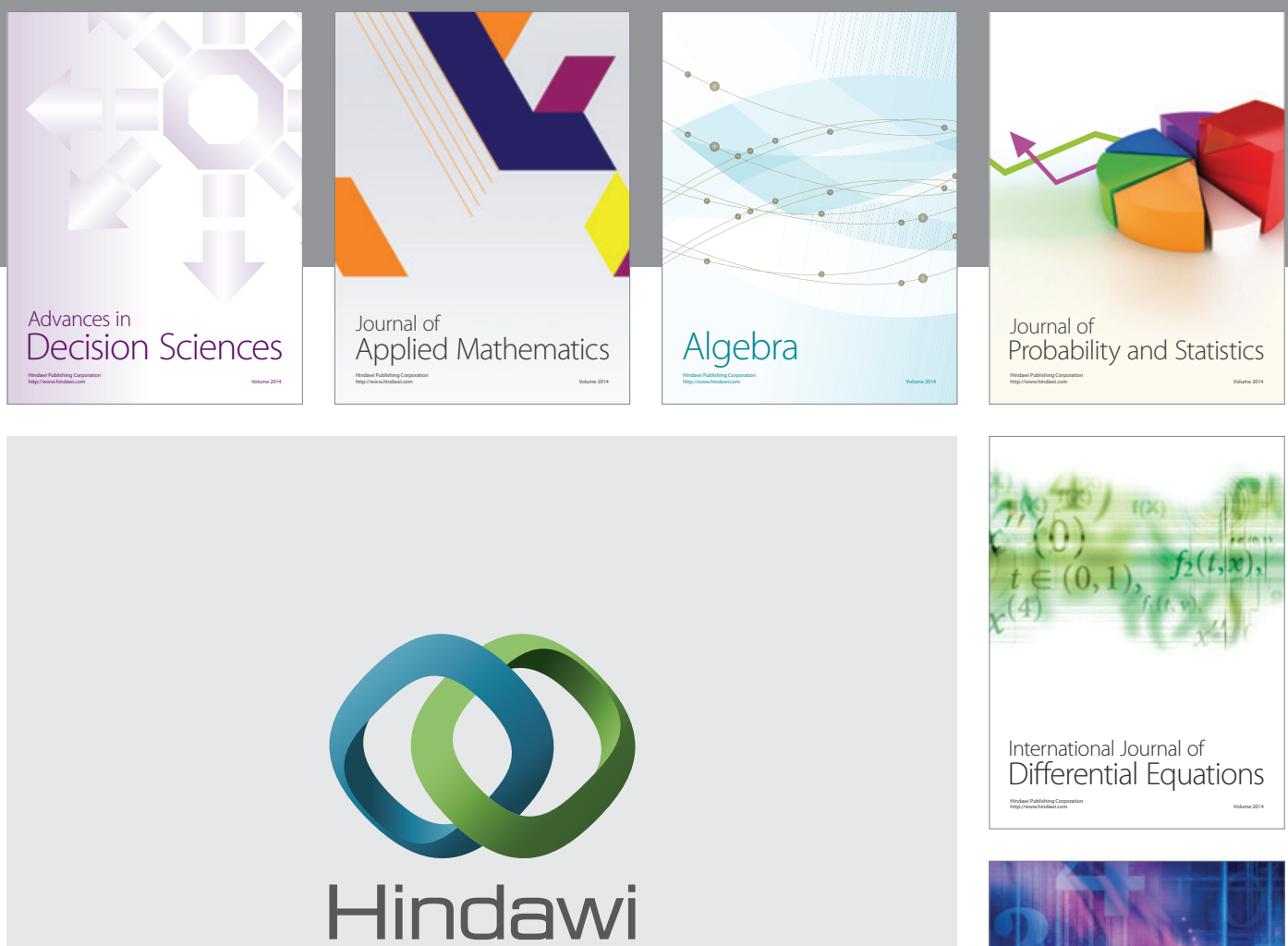

Submit your manuscripts at http://www.hindawi.com
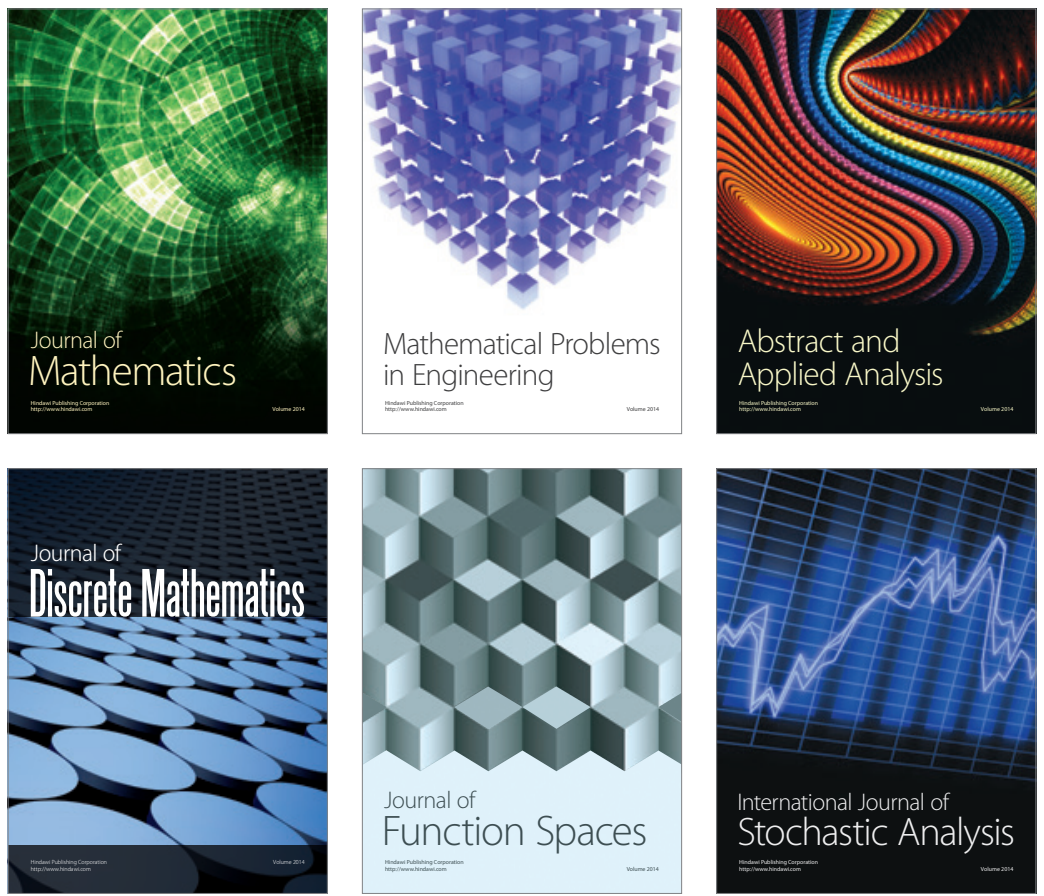

Journal of

Function Spaces

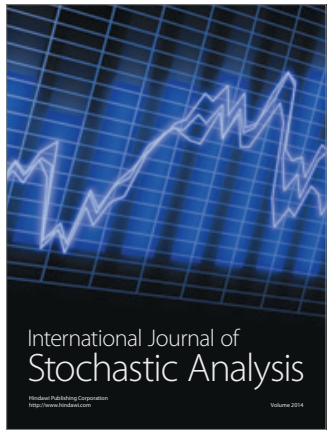

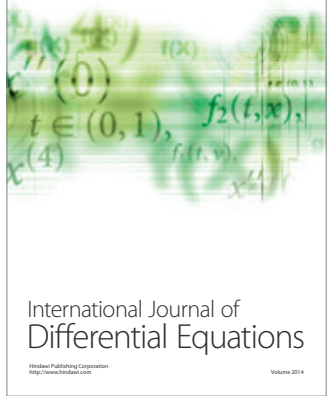
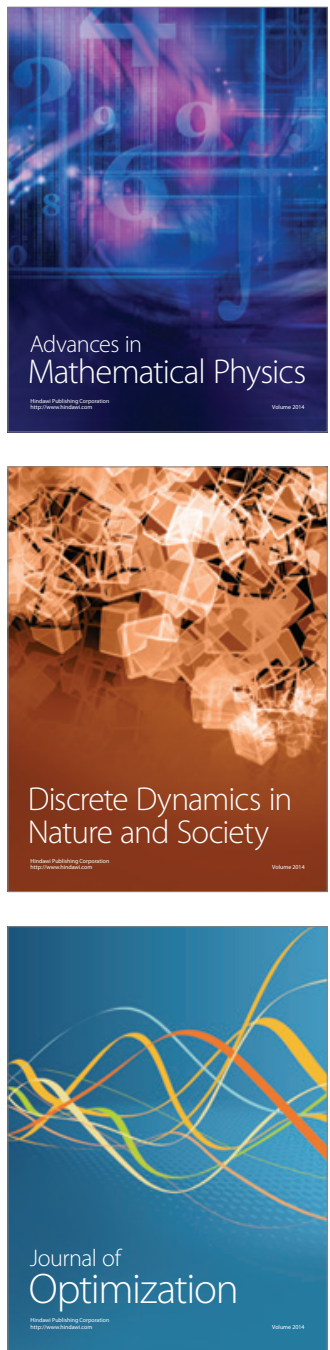